\title{
Rizobacteria: in plants mitigates the water deficit
}

\begin{abstract}
Plant water deficit, causes losses in all stages of development and productivity, plants have mechanisms to minimize the negative effects of this stress, studies with plant growth promoting rhizobacteria (PGPR) are able to act to mitigate damage in plants. plants, by the protection of the photosynthetic apparatus, with an increase in carotenoid concentration, reducing photochemical damage, as well as helping to change the concentration of osmoregulators in plants, such as proline and malonic aldehyde (MDA), and eliminating reactive oxygen species ( ROS).
\end{abstract}

Keywords: PGPR, biochemistry, osmoregulator
Volume 7 Issue 3 - 2018

\author{
Marcela Cristiane Ferreira Rêgo, Gleiciane \\ Rodrigues dos Santos, Gisele Barata da Silva \\ Doctoral program in agronomy of the university federal rural of \\ amazonia, Brazil
}

\begin{abstract}
Correspondence: Marcela Cristiane Ferreira Rêgo, Doctoral program in agronomy of the university federal rural of amazonia, Brazil, Email marcela.c.f.rego@ufra.edu.br
\end{abstract}

Received: April II, 2018 | Published: May 31, 2018
Abbreviations: PGPR, plant growth promoter rhizobacteria; MDA, malonic aldehyde; ROS, reactive oxygen species; ISR, induced defense system; $\mathrm{Ci}$, internal carbon

\section{Introduction}

The abiotic stresses (water stress, saline, temperature) in which plants are submitted during their cultivation cycle induce plants to biochemical responses, which act in the induced defense system (ISR). And studies in bioinoculant plants with growth promoting rhizobacteria (PGPR) show that the response to mitigation of damage to abiotic stresses is potentiated by plants.

About 5 to $21 \%$ of the internal carbon (Ci) fixed in the leaves is transported to the roots and released into the rhizosphere through root exudation, which together with the exopolysaccharides (released by the PGPR) act in the formation of a biofilm, which aid in attraction and bacterial fixation on the epidermis of the roots, which then penetration and colonization along the root.

Our studies with Burkholderia pyrrocinia BRM-32113 and Pseudomonas fluorescens BRM-32111, in upland rice plants over water deficit, were able to mitigate the damage caused by the déficit (Figure 1)
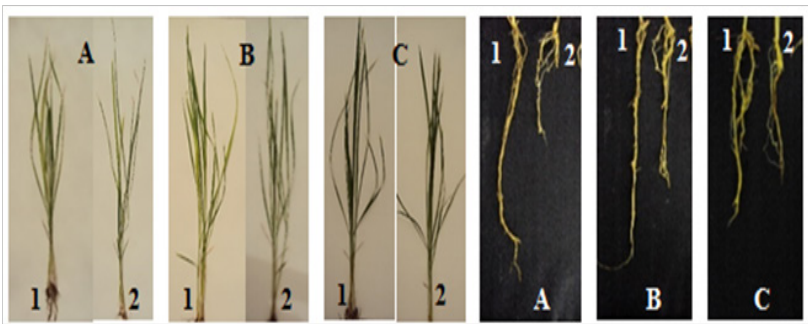

Figure I Leaf and roots of rice plants, from seeds inoculated with PGPR and subjected to water deficit. Rice plants uninoculated (control) $(A), B$. pyrrocinia BRM-32II 3 (B) and P. fluorescens BRM-32III (C), in I00\% field capacity (I) and $50 \%$ field capacity (2).

\section{PGPR action on plants in water deficit}

PGPRs induce increased production of carotenoids and chlorophyll $\mathrm{b}$ (accessory pigments) that absorb photons and transfer excitation energy to reaction centers via chlorophyll, and protect chlorophyll from photo-oxidation, carotenoids also act as antioxidants and against photochemical damage.

The loss of chlorophyll content under water stress is considered one of the main causes of photosynthesis decrease. In addition, the reduction of chlorophyll content can be attributed to loss of chloroplast membranes through the reactions of water-soluble enzymes located in the chloroplast stroma (Calvin cycle) or at the border between stroma and thylakoids (ATP-ase, $\mathrm{NO}_{2}$, and reduction of $\mathrm{NADP}^{+}$) that are affected by osmotic stress. ${ }^{1}$

\section{Induction of accumulation of osmoregulators by prgr}

The highest accumulation of proline in plants occurs as a response to water deficit tolerance induced by PGPR, which in plants without PGPR, ${ }^{2}$ as shown in plant studies under stress: water deficit, salinity, low temperature, exposure to heavy metals, UV radiations. Proline is a highly soluble, low molecular weight, organic compound and generally nontoxic at high cell concentrations and acts on cellular osmotic adjustment and detoxification of reactive oxygen species (ROS), protection of membrane integrity, enzymes and stabilization of proteins. ${ }^{3}$

In membranes and proteins, elimination of free radicals and redox cellular buffer under stress conditions occurs and can also act to alleviate cytoplasmic acidosis and maintain $\mathrm{NADP}^{+} / \mathrm{NADPH}$ compatible with metabolism. Its accumulation normally occurs in the cytoplasm where it functions as molecular chaperons stabilizing the structure of the proteins and their buffers for cytosolic $\mathrm{pH}$ accumulation and maintains the cellular redox state. ${ }^{4}$

The increase in the accumulation of MDA is an indicator of oxidative damage in plants under stress conditions, in plants inoculated with PGPR less accumulation of MDA resulting in the protection 
of the membranes, since the high concentration of MDA produced during lipid peroxidation, a strong indicator of cell damage caused to the membrane which acts on decreasing the membrane stability that reflects the extent of lipid peroxidation.

Lipid peroxidation is an indicator of the free radical reaction in tissues, where oxygen uptake in tissues generates reactive oxygen species (ROS), particularly $\mathrm{H}_{2} \mathrm{O}_{2}$, which is produced at high rates by the glycolate oxidase in the peroxisomes in photorespiration. ${ }^{5}$

\section{Conclusion}

In this way, PGPRs act in the process of mitigating the damages caused by the water deficit in the plants, by maintaining the integrity of enzymes and proteins of cell wall, and in the increase of osmoregulators, acting in the osmotic equilibrium of the system.

\section{Acknowledgements}

Doctoral program in agronomy of the university federal rural of amazonia.

\section{Conflict of interest}

The author declares that there is no conflict of interest.

\section{References}

1. Guerfel M, Baccouri O, Boujnah D, et al. Impacts of water stress on gas exchange, water relations, chlorophyll content and leaf structure in the two main Tunisian olive (Olea europaea L.) cultivars. Sci Horticult. 2009;119(3):257-263.

2. Serraj R, Sinclair TR. Osmolyte accumulation: can it really help increase crop yield under drought conditions? Plant Cell Environ. 2002;25(2):333341 .

3. Rhodes D, Nadolska-Orczyk A, Rich PJ. Salinity, osmolytes and compatible solutes. In: Lauchli A, Luttge U, editors. Salinity, Environment, Plant, Molecules. Netherlands: Al-Kluwer Academic Publishers; 2002:181-204.

4. Ashraf M, Foolad MR. Roles of glycine betaine and proline in improving plant abiotic stress resistance. Environmental and Experimental Botany. 2007;59(2):206-216.

5. Yang F, Miao LF. Adaptive responses to progressive drought stress in two poplar species originating from different altitudes. Silva Fennica. 2010;4:23-37. 